\title{
Exit interaction effect on nascent product state distribution of $\mathrm{O}(\mathrm{D}$ - 1) $+\mathrm{N} 2 \mathrm{O} \rightarrow \mathrm{NO}+\mathrm{NO}$
}

$\operatorname{AUTHOR}(\mathrm{S}):$

Kawai, S; Fujimura, Y; Kajimoto, O; Takayanagi, T

\section{CITATION:}

Kawai, S ... [et al]. Exit interaction effect on nascent product state distribution of O(D1)+N2O $\rightarrow$ NO+NO. JOURNAL OF CHEMICAL PHYSICS 2004, 120(14): 6430-6438

\section{ISSUE DATE:}

2004-04-08

URL:

http://hdl.handle.net/2433/49844

\section{RIGHT:}

Copyright 2004 American Institute of Physics. This article may be downloaded for personal use only. Any other use requires prior permission of the author and the American Institute of Physics. 


\title{
Exit interaction effect on nascent product state distribution of $\mathrm{O}\left({ }^{1} \mathrm{D}\right)+\mathrm{N}_{\mathbf{2}} \mathrm{O} \rightarrow \mathrm{NO}+\mathrm{NO}$
}

\author{
Shinnosuke Kawai, Yo Fujimura, and Okitsugu Kajimoto \\ Department of Chemistry, Graduate School of Science, Kyoto University, Kitashirakawa-Oiwakecho, \\ Sakyo-ku, Kyoto 606-8502, Japan \\ Toshiyuki Takayanagi \\ Advanced Science Research Center, Japan Atomic Energy Research Institute, Tokaimura, Naka-gun, \\ Ibaraki 319-1195, Japan
}

(Received 24 November 2003; accepted 30 December 2003)

\begin{abstract}
We have determined the rotational state distributions of $\mathrm{NO}\left(v^{\prime}=0,1,2\right)$ products produced from the reaction $\mathrm{O}\left({ }^{1} \mathrm{D}\right)+\mathrm{N}_{2} \mathrm{O}$. This is the first full characterization of the product rotational distribution of this reaction. The main part of each rotational distribution (up to $j^{\prime} \approx 80$ ) has rotational temperature $\approx 20000 \mathrm{~K}$ and all these distributions are quite near to those predicted by the phase space theory (PST). This observation and previously reported vibrational distribution indicate that the most part of the energy partitioning of the reaction products is at least apparently statistical although the intermediate of this reaction is not so stable as to ensure the long lifetime. On the other hand, the distributions in the high rotational levels $\left(j^{\prime}=80-100\right)$ are found to decrease more sharply as $j^{\prime}$ increases than the PST predictions. The origin of the observed decrease of the distribution is discussed with quasiclassical trajectory (QCT) calculations on a five-dimensional $a b$ initio potential energy surface (PES). The observed near-statistical distribution and the sharp decrease in the high$j^{\prime}$ levels are well reproduced by a "half-collision" QCT calculation, where statistical distribution at the reaction intermediate is assumed. This agreement shows the rotation-translation interaction in the exit region has an effect of yielding small high- $j^{\prime}$ populations. However, a little bias of the calculated distribution toward lower rotational excitation than the observed one indicates that the combination of the statistical intermediate and the exit interaction on the current PES does not completely describe the real system. It is suggested that the reaction intermediate is generated with the distribution which is close to statistical but a little biased toward yielding high- $j^{\prime}$ products, and that the interaction in the exit region of the PES results in the sharp decrease in the high- $j^{\prime}$ levels.

(C) 2004 American Institute of Physics. [DOI: 10.1063/1.1649721]
\end{abstract}

\section{INTRODUCTION}

The reaction of $\mathrm{O}\left({ }^{1} \mathrm{D}\right)$ with $\mathrm{N}_{2} \mathrm{O}$ has two major channels:

$\mathrm{O}\left({ }^{1} \mathrm{D}\right)+\mathrm{N}_{2} \mathrm{O} \rightarrow \mathrm{NO}+\mathrm{NO}, \quad \Delta H_{\mathrm{f}}^{\mathrm{o}}(0)=-341 \mathrm{~kJ} / \mathrm{mol}$,

$\mathrm{O}\left({ }^{1} \mathrm{D}\right)+\mathrm{N}_{2} \mathrm{O} \rightarrow \mathrm{N}_{2}+\mathrm{O}_{2}, \quad \Delta H_{\mathrm{f}}^{\mathrm{o}}(0)=-520 \mathrm{~kJ} / \mathrm{mol}$.

These two channels can be characterized by large exothermicity ${ }^{1}$ and almost gas kinetic rate constants. ${ }^{2}$ The branching ratio of the two channels was experimentally determined to be $k_{1} / k_{2}=1.6$ although there has been no observation which identifies the electronic state of the nascent $\mathrm{O}_{2}$ products for channel (2). Most studies of this reaction were concerned with the channel (1) because of the ease of detection of NO products and its importance in atmospheric chemistry as a major source of stratospheric NO.

A peculiar feature of the dynamics of this reaction is the release of the large exothermicity without a deep potential well. For channel (1), NO dimer is the known stable species. There are large amounts of spectroscopic study on the cisplanar dimer and its binding energy was determined to be about $8.5 \mathrm{~kJ} / \mathrm{mol},{ }^{3}$ which is very small compared to the exo- thermicity of $341 \mathrm{~kJ} / \mathrm{mol}$. Ab initio calculations ${ }^{4-8}$ found no deeper well than the cis-planar ONNO and showed that this channel has almost no barrier. The very shallow well suggests that the lifetime of the intermediate of this reaction is short.

The dynamics of the channel (1) has been studied by measuring product state distributions and vector properties, ${ }^{9-17}$ mostly based on the laser-induced fluorescence (LIF) technique. The two NO molecules produced from this reaction should be distinguished from dynamical viewpoint. The newly formed $\mathrm{NO}$ originating from $\mathrm{O}\left({ }^{1} \mathrm{D}\right)$ is called a "new" NO and the other NO which already exists in the reactant $\mathrm{N}_{2} \mathrm{O}$ is called an "old" $\mathrm{NO}$. Using isotopically labeled ${ }^{18} \mathrm{O}\left({ }^{1} \mathrm{D}\right)$, Akagi et al. ${ }^{13,14}$ measured the individual vibrational state distributions of the new and old NO products for $v^{\prime} \leqslant 17$ in a condition where the nascent rotational distributions are relaxed by collisions after the reaction but vibrational relaxation is negligible. The distribution decreased monotonically as the vibrational quantum number increased and significant populations were found up to $v^{\prime}$ $\approx 10$. The sum of the distribution of the new and old NO products was very close to the statistical distribution up to $v^{\prime} \approx 10$ and became smaller than the statistical one for higher 
$v^{\prime}$. The vibrational distributions measured with Fourier transform infrared (FTIR) spectrometer ${ }^{12,17}$ showed better agreement with the statistical one for such high $v^{\prime}$ levels. Furthermore, although the vibrational distribution of the new $\mathrm{NO}$ is more excited than that of the old NO, the populations of both types of the products in each vibrational level are not significantly different. ${ }^{13,14}$ This result forms a sharp contrast to the $\mathrm{O}\left({ }^{1} \mathrm{D}\right)+\mathrm{H}_{2} \mathrm{O}$ reaction, whose two types of $\mathrm{OH}$ products show entirely different vibrational distributions ${ }^{18,19}$ although the reaction is considered to proceed with stable $\mathrm{H}_{2} \mathrm{O}_{2}$ intermediate. ${ }^{20}$ Akagi et al. compared the observed vibrational distribution with those for $\mathrm{O}\left({ }^{1} \mathrm{D}\right)+\mathrm{H}_{2} \mathrm{O}$ and $\mathrm{S}\left({ }^{1} \mathrm{D}\right)+\mathrm{N}_{2} \mathrm{O} \cdot{ }^{21}$ From the analysis of vibrational couplings, they concluded that the existence of atoms with similar masses induced enhancement of the energy transfer, which compensated the short lifetime of the intermediate.

For rotational distribution, significant excitation of the NO products was first suggested by Goldstein et al. ${ }^{22}$ They observed congested rotational lines in the $\Delta v=0$ region of the NO $A^{2} \Sigma^{+}-X^{2} \Pi$ transition but did not make any quantitative analysis. As opposed to this observation, Brouard et al. reported a cold distribution of $\mathrm{NO}\left(v^{\prime}=0\right)$ almost identical to a thermal distribution of $300 \mathrm{~K} .^{10,11}$ They found high rotational excitation for $\mathrm{NO}\left(v^{\prime} \geqslant 1\right)$ and thus they suggested that $\mathrm{NO}\left(v^{\prime}=0\right)$ was produced via stripping mechanism whereas $\mathrm{NO}\left(v^{\prime} \geqslant 1\right)$ was produced through a short-lived complex. On the other hand, Tsurumaki et al. ${ }^{15}$ found that the rotational distribution of $\mathrm{NO}\left(v^{\prime}=0\right)$ was much more excited and estimated the rotational temperature to be $T_{\text {rot }}$ $>10000 \mathrm{~K}$, which is also close to the statistical distribution. They suggested the possibility that the thermalized residual NO contaminated the spectrum in the measurements of Brouard et al. ${ }^{10,11}$ The near-statistical rotational distribution observed by Tsurumaki et al. ${ }^{15}$ is also indicative of efficient energy transfer within the intermediate. However, one cannot conclude that the rotational distribution of $\mathrm{NO}\left(v^{\prime}=0\right)$ is really near-statistical since their measurement is limited to $j^{\prime}<50$, which covers only the half of the rotational populations in $v^{\prime}=0$ level if the statistical or the Boltzmann distribution continues for all the higher rotational levels. The reason for this limitation is the overlap of the transitions from high- $j^{\prime}$ levels of $(0,0)$ band with $(1,1)$ and $(2,2)$ bands of the $A-X$ transition. Due to the widely spread rovibrational distribution of NO products, such overlap makes the spectral assignment severely difficult.

In theoretical works, it is only quite recent that this reaction was treated as a four-atomic system. González et al. ${ }^{5}$ calculated the potential energies at the stationary points and obtained the rate constants using transition state theory and quasiclassical trajectory (QCT) calculation on a fitted pseudotriatomic London-Eyring-Polanyi-Sato surface. Although they found relatively good agreement with the experimental results in the rate constants and branching ratios, their pseudotriatomic surface was not adequate to calculate the product state distributions. Takayanagi et al. ${ }^{6-8}$ calculated the potential energies at about 10000 grid points of planar configurations of this system and fitted them to an analytical form. Using this ${ }^{1} A^{\prime}$ ground surface, they performed QCT calculation with zero impact parameter ${ }^{8}$ and quantum calculations. ${ }^{7,23}$ They found qualitative agreement with the experimental results on vibrational distribution and branching ratio. However, the rotational distributions were not calculated.

In this paper, we have fully determined the rotational state distributions of $\mathrm{NO}\left(v^{\prime}=0,1,2\right)$ products by measuring the wide range of LIF spectra (up to $j^{\prime} \approx 100$ ) and by carefully assigning the large number of rotational lines. It is found out that the overall trend of each rotational state distribution is close to statistical. However, the distribution decreases more rapidly as $j^{\prime}$ increases than the statistical distribution in high rotational levels $j^{\prime}>80$ for all these vibrational levels. Furthermore, we carry out QCT calculations on the $a b$ initio potential energy surface (PES) calculated by Takayanagi et al. to predict rotational distributions in these vibrational levels. In addition to ordinary QCT calculations, we perform another type of calculation which consists of statistical distribution of the reaction intermediate and usual propagation to the products. This calculation enables us to separately extract the effects of the PES from the reactant to the intermediate and from the intermediate to the product, to provide us with deeper insight into the dynamics. The details of the dynamics of the reaction will be discussed based on the comparison between the experimental and theoretical results, especially in terms of the effect of the exit region of the PES from the intermediate to the product.

\section{EXPERIMENT}

The experiment was performed in flow condition at room temperature. Nitrous oxide (Showa Denko 99.999\%) was used without further purification. The sample gas was introduced into a stainless steel vacuum chamber and pumped with a rotary pump and a mechanical booster pump. The pressure was kept at 200 mTorr by controlling the flow rate with a stainless steel needle valve.

The electronically excited oxygen atom $\mathrm{O}\left({ }^{1} \mathrm{D}\right)$ was produced from the photodissociation of $\mathrm{N}_{2} \mathrm{O}$ with $193 \mathrm{~nm}$ light generated by an ArF excimer laser (Lambda Physik EMG 53 MSC). The product $\mathrm{NO}\left(v^{\prime}=0,1,2\right)$ molecules were probed with LIF via the NO $A^{2} \Sigma^{+}-X^{2} \Pi(0,0)(0,1)(0,2)$ transitions. To obtain the probe light, a tunable dye laser (Lambda Physik SCANmate 2E) pumped by a $\mathrm{XeCl}$ excimer laser (Lambda Physik COMPex 102) was utilized. The wavelength of the probe light was $213-248 \mathrm{~nm}$ and it was generated by using the laser dyes Stilbene $3(213-216 \mathrm{~nm}$, as the doubled output), Coumarin 120 (215-222 nm), Coumarin 2 (220-228 nm), Coumarin $47(226-239 \mathrm{~nm})$, and Coumarin $102(239-248 \mathrm{~nm})$ and by frequency doubling their output with BBO I (220-248 nm) and BBO II (213-221 nm) crystals. The delay time between the photolysis and the probe lights was set to be $100 \pm 20 \mathrm{~ns}$, which has been proved to be sufficient to neglect the effect of collisional quenching of the nascent distribution. ${ }^{15}$ In order to minimize the saturation effect, the intensity of the probe laser light was kept about 7-8 $\mu \mathrm{J} /$ pulse and the dependence of the LIF signal on the intensity of the probe light was measured in advance. The photolysis and the probe lights were counterpropagated with each other. 
The LIF signal was collected by a synthetic silica lens and focused by another silica lens on a photomultiplier tube (Hamamatsu R928) mounted in the direction perpendicular to the laser axis. In order to reduce the strong scattered light and emission arising from the photolysis laser, a long-pass filter (Corning CS9-54) was placed in front of the photomultiplier tube. The detected signal was amplified by a fast preamplifier (Comlinear CLC100) and gated by a boxcar integrator (Stanford Research System SR250). The first 50 ns after the probe laser irradiation was not included in the integration to avoid the scattering of the probe light. The gate width was set to $100 \mathrm{~ns}$ in consideration of the fluorescence lifetime. The gated signal was digitized by an A/D converter (Stanford Research System SR245) and stored into a personal computer via a GPIB interface. The intensities of the photolysis and the probe lights were monitored with photodiodes (Hamamatsu 1336-5BQ) to normalize the detected LIF signals. The absolute intensities of the probe laser were also measured with laser power meter (Gentec ED-100A). The experimental system was operated with a repetition rate of $10 \mathrm{~Hz}$.

As reported previously, ${ }^{15}$ there exist thermalized residual NO molecules in the reaction chamber. The contribution of the residual NO to the LIF signal is considerable in the low $j^{\prime}$ levels only for $v^{\prime}=0$ level. In order to eliminate this background signal, we altered the ON/OFF of the photolysis laser shot by shot and subtracted the signal without the photolysis laser from that with the photolysis laser.

Recent study of Adams et al. detected a spin-forbidden $\mathrm{N}\left({ }^{4} \mathrm{~S}\right)+\mathrm{NO}$ channel ${ }^{24}$ although the yield was not expected to exceed $1 \% .{ }^{25}$ By examining the dependence of the LIF signal intensity on the delay time, we confirmed the negligible contribution of the photofragment NO, which would arise just after the photolysis.

Since $\mathrm{NO}\left(A^{2} \Sigma^{+}\right)$is known to predissociate, ${ }^{26}$ we checked the time profile of the fluorescence. The LIF signals of 16 rotational levels were recorded in a digital storage oscilloscope (Tektronix TDS3052B).

\section{QUASICLASSICAL TRAJECTORY CALCULATION}

In order to understand the origin of observed rotational populations, we have preformed QCT calculations. We used the PES of Takayanagi et al. ${ }^{6-8}$ which is an analytically fitted surface of $a b$ initio calculations of the lowest ${ }^{1} A^{\prime}$ PES. The details of the PES can be found in Refs. 6-8.

The classical equations of motion for the $\mathrm{O}\left({ }^{1} \mathrm{D}\right)+\mathrm{N}_{2} \mathrm{O}$ collision were numerically integrated by using BulirschStoer method. ${ }^{27}$ Energy conservation up to the order of 0.01 $\mathrm{cm}^{-1}$ was confirmed. For the coordinate, three Jacobi vectors were employed; one connects the $\mathrm{O}\left({ }^{1} \mathrm{D}\right)$ atom and the center of mass $(\mathrm{CM})$ of the $\mathrm{N}_{2} \mathrm{O}$ molecule, another connects the terminal $\mathrm{N}$ atom and $\mathrm{CM}$ of $\mathrm{NO}$ moiety in $\mathrm{N}_{2} \mathrm{O}$, and the other connects the $\mathrm{N}$ and $\mathrm{O}$ atoms of $\mathrm{NO}$ in $\mathrm{N}_{2} \mathrm{O}$. Since the $a b$ initio calculations were performed only for the planar structure of the $\mathrm{O}\left({ }^{1} \mathrm{D}\right)+\mathrm{N}_{2} \mathrm{O}$ system, the three Jacobi vectors were confined in two-dimensional (2D) collision plane. The calculated population of each rotational state was multiplied by the degeneracy factor $2 j^{\prime}+1$ to convert the $2 \mathrm{D}$ results to 3D. This conversion is on the assumption that all the $2 j^{\prime}+1$ states are populated equally. We consider this assumption valid because the anisotropy of the product rotational angular momentum in the $\mathrm{CM}$ frame was found to be small ${ }^{15}$ and, as will be shown in Sec. IV A, we have found only slight preference for one of the $\Lambda$-type doublet components to the other.

To obtain detailed insight into the dynamics, we have carried out two types of calculations. One is the conventional classical scattering calculation, which we call full-collision calculation. The other type is named half-collision calculation. In the latter calculation, we have assumed a statistical distribution in the reaction intermediate ONNO and then observed the resulting NO state distributions. More precisely, phase space points with fixed distance between the CM of each NO were randomly sampled and trajectories were propagated en route to the products. For this sampling, the distance between the CM of two NO's was chosen equal to the experimentally determined value of NO dimer, i.e., $2.263 \AA{ }^{28}$

For the full-collision calculation, collision energy was sampled according to the distribution of collision energy in the present experimental condition. The distribution was calculated from the convolution of the velocity distribution of $\mathrm{O}\left({ }^{1} \mathrm{D}\right)$ and that of thermal $\mathrm{N}_{2} \mathrm{O}$ by taking account of the integration of the angle between these two velocity vectors. ${ }^{29}$ For the velocity distribution of $\mathrm{O}\left({ }^{1} \mathrm{D}\right)$, experimentally determined translational energy distribution for the $193 \mathrm{~nm}$ photodissociation of $\mathrm{N}_{2} \mathrm{O}$ (Ref. 30) was used. The mean collision energy $\left\langle E_{\text {col }}\right\rangle$ thus obtained is $52 \mathrm{~kJ} / \mathrm{mol}$ and the full width at half maximum of the $E_{\mathrm{col}}$ distribution is $38 \mathrm{~kJ} / \mathrm{mol}$. The initial rotational energy of $\mathrm{N}_{2} \mathrm{O}$ was sampled by classical Boltzmann distribution of $300 \mathrm{~K}$ and the vibrational energy was fixed to the zero point energy of the calculated PES. Impact parameters were sampled up to $6 \AA$, where the opacity function became negligibly small. For the halfcollision calculation, the total energy of the system was set equal to the collision energy of $\mathrm{O}\left({ }^{1} \mathrm{D}\right)$ and $\mathrm{N}_{2} \mathrm{O}$ plus the zero point energy of $\mathrm{N}_{2} \mathrm{O}$. About 200000 trajectories were run for each of the two types of the calculation.

\section{RESULTS AND DISCUSSIONS}

\section{A. Experimental results}

The entire LIF spectrum measured in this study is exhibited in Fig. 1. The spectral regions of vibronic bands are shown with their vibrational quantum numbers. Due to the high excitation of $\mathrm{NO}$ products in rotational and vibrational motions, a large number of peaks appeared in the spectrum and some of them overlapped with each other. We carefully assigned all the peaks by using the spectroscopic constants found in Ref. 31. Only those peaks which do not overlap with other peaks were utilized to determine the vibrational and rotational distribution. In Fig. 1, peaks from wide range of $j^{\prime}$ were observed. Hence, in order to properly derive the population from the LIF spectrum, we had to carefully check the experimental conditions and correct the observed signals accordingly.

First, to examine the effect of saturation, we measured the dependence of the LIF signal on the dye laser power. 


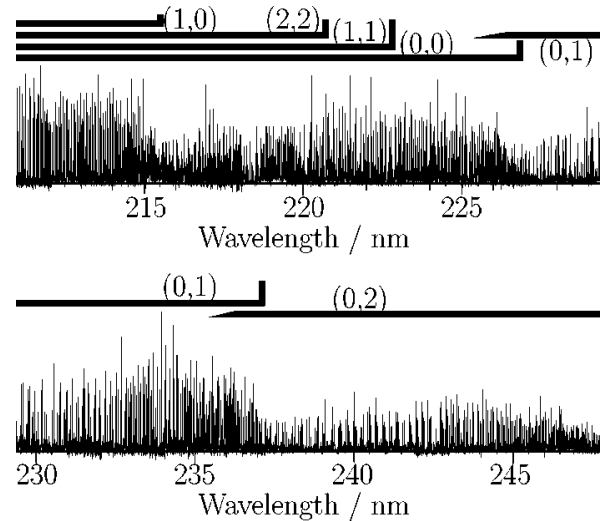

FIG. 1. Observed LIF spectra of nascent $\mathrm{NO}\left({ }^{2} \Pi_{1 / 2,3 / 2}\right)$. The spectral regions are roughly shown for each vibronic band with upper and lower state vibrational quantum numbers.

Typical results are shown in Fig. 2. It is known that the intensity $I_{\mathrm{LIF}}$ of LIF signals is proportional to $P[1$ $-\exp [-(A+2 B \rho) \Delta t]$ when the rate equation for the twostate model is solved to take into account the saturation effect. ${ }^{32}$ Here, $P$ denotes the population in a specific quantum state, $A$ and $B$ are the Einstein coefficients, $\rho$ expresses the radiation density, and $\Delta t$ is the duration of the laser pulse assumed as rectangular. As shown in Fig. 2, this expression can be effectively approximated as $C(\rho S)^{n} P$ and we fitted $I_{\mathrm{LIF}}$ to this formula. Here, $C$ and $n$ are fitting parameters, and $S$ denotes the line strength factor. We found $n=0.8-1.0$ by least-squares fitting and confirmed that the derived distribution did not change significantly in this range of $n$. We adopted $n=0.9$ for all the rotational lines to determine the population and Fig. 1 shows the spectrum after the correction against the probe laser intensities with this value. To derive rotational distributions, we divided the signal intensity in the
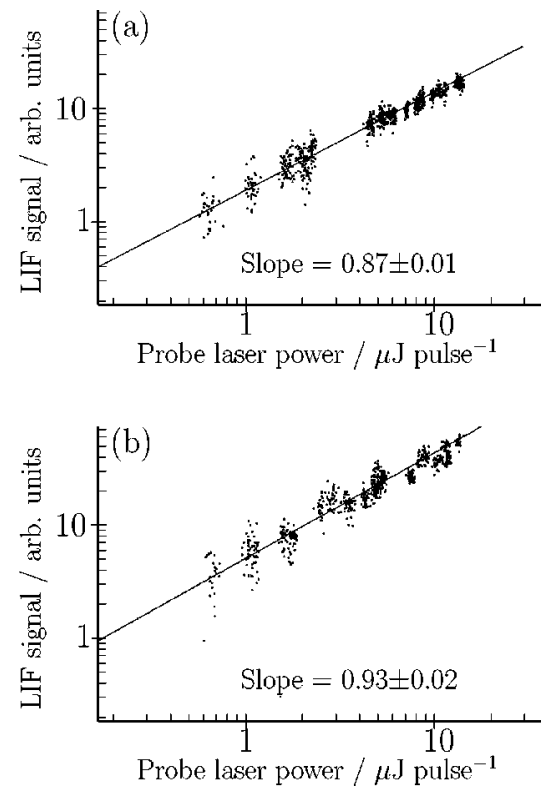

FIG. 2. Dependence of the LIF signal intensities on the probe dye laser powers measured for (a) $Q_{11}(47.5)$ and (b) $Q_{22}(67.5)$ transitions of $A-X(0,0)$ band. The solid lines represent the results of least-squares fitting and their slopes are shown.

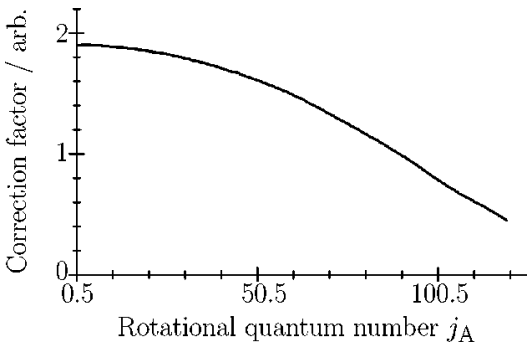

FIG. 3. Correction factors for the LIF signal whose upper state is $\mathrm{NO}\left(A, v_{A}=0, j_{A}\right)$ calculated from Franck-Condon factors, Hönl-London factors, filter transmission, and photomultiplier sensitivity.

spectrum by $S^{n}$, where $S$ was calculated by taking into account the Hönl-London factors and the dependence of electronic transition moment on rotational levels. ${ }^{33}$ The correction due to the electronic transition moment decreases the line strength factor for $j^{\prime} \approx 100$ by at most $20 \%$ compared to that for $j^{\prime} \approx 0$.

Second, signal intensity was corrected against the wavelength dependence of the detection efficiency since the difference of the wavelengths of the fluorescence from the low and high rotational levels was significant. We calculated the wavelength dependence of the detection efficiency from the curves of the filter transmission and the photomultiplier sensitivity, which were taken from their catalogs. With thus obtained efficiency curve, the correction factors for the LIF detection was determined by considering Franck-Condon factors $^{31}$ and Hönl-London factors. The resulting correction factors for $\operatorname{NO}\left(A, v_{A}=0, j_{A}\right)$ are shown in Fig. 3. The detection efficiency monotonically decreases with $j_{A}$ and that of $j_{A}=0.5$ is 2.5 times higher than that of $j_{A} \approx 100$.

Third, the rotational state dependence of the predissociation rate should be taken into account. $\operatorname{NO}\left(A, v_{A}\right)$ is known to predissociate in $v_{A}>3$ levels. ${ }^{26}$ The threshold energy corresponds to $j_{A} \approx 65$ for $\mathrm{NO}\left(A, v_{A}=0\right)$ although the dissociation rate has not been reported for such high rotational levels. To clarify the appropriate correction factor, we measured the time profiles of the LIF signals from $\operatorname{NO}\left(A, v_{A}=0, j_{A}\right)$. The time constants of the fluorescence decay were determined from least-squares-fitting analysis of the recorded profiles with the assumption of single exponential decay. Figure 4 shows the lifetimes against the upper state rotational quantum number $j_{A}$. As recognized from the figure, clear dependences on $j_{A}$ are not found although the lifetimes are scattered. Thus, no further correction due to the predissociation was made for determining the populations. Although this de-

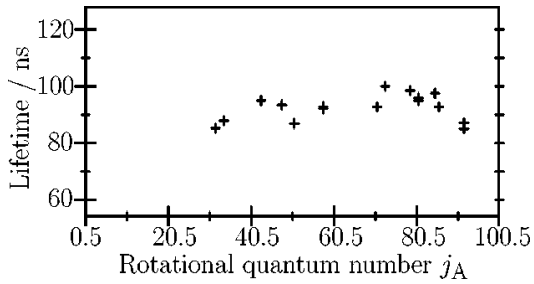

FIG. 4. Fluorescence lifetime of $\operatorname{NO}\left(A^{2} \Sigma^{+}, v_{A}=0, j_{A}\right)$ measured with 200 mTorr $\mathrm{N}_{2} \mathrm{O}$. No significant dependence on either $j_{A}$ or spin-rotation sublevels was found. 

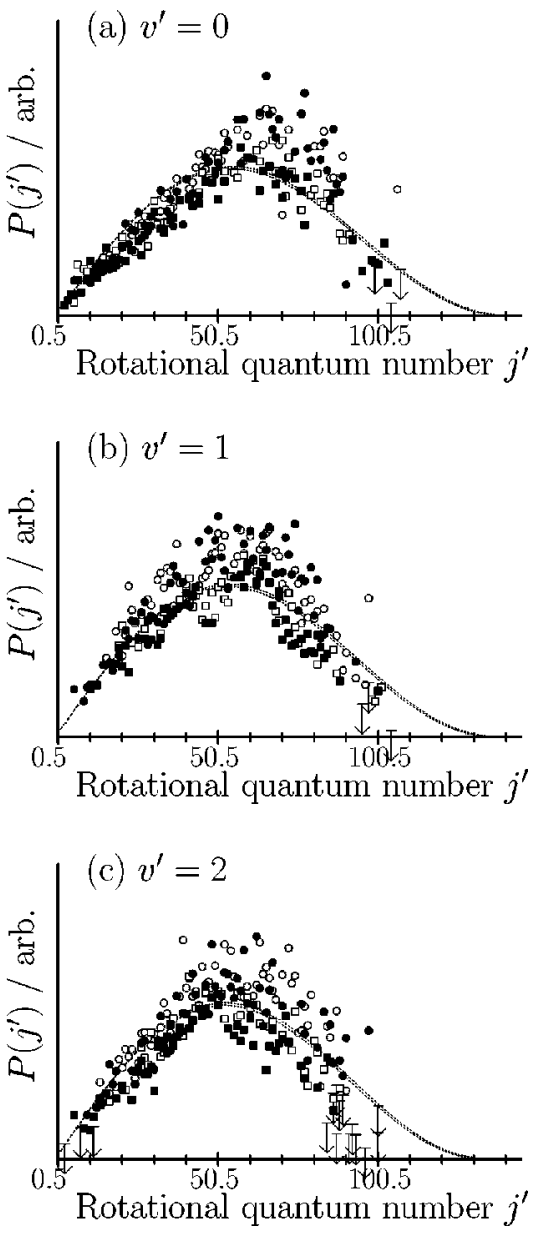

FIG. 5. Rotational state distributions of $\mathrm{NO}\left(v^{\prime}=0,1,2\right)$. Squares and circles denote the observed populations $\left(\bigcirc,{ }^{2} \Pi_{1 / 2} A^{\prime} ; \square,{ }^{2} \Pi_{1 / 2} A^{\prime \prime} ; \boldsymbol{\bullet},{ }^{2} \Pi_{3 / 2} A^{\prime} ; \mathbf{\square}\right.$, $\left.{ }^{2} \Pi_{3 / 2} A^{\prime \prime}\right)$. Downward arrows show upper limits estimated from noise levels. Dotted lines are the rotational state distributions of ${ }^{2} \Pi_{1 / 2}$ and ${ }^{2} \Pi_{3 / 2}$ levels calculated by using PST.

pendence is not a central topic of this paper, it is notable that the effects of the rotational energy and vibrational energy are different for the predissociation of $\mathrm{NO}(A)$.

The observed lifetimes ranging from 80 to $100 \mathrm{~ns}$ are shorter than the collision-free radiative lifetime of $\operatorname{NO}(A)$, i.e., $200 \mathrm{~ns}^{26}$ This result indicates that the rate constant of electronic quenching is about $(200 \mathrm{~ns})^{-1}$ with $200 \mathrm{mTorr}$ of $\mathrm{N}_{2} \mathrm{O}$, which corresponds to $\approx 8 \times 10^{-10} \mathrm{~cm}^{3}$ molecule ${ }^{-1} \mathrm{~s}^{-1}$. It may imply that the products can suffer collisions in the time scale of our pump-probe delay under the present pressure. However, the known rate constants of rotational relaxation of $\mathrm{NO}$ with diatomic and triatomic species $^{34}$ are smaller than the electronic quenching rate found here and even if we use this electronic quenching rate as the upper limit of the rotational quenching, at least $80 \%$ of $\mathrm{NO}\left(X, v^{\prime}, j^{\prime}\right)$ products generated during the $100 \mathrm{~ns}$ delay time is estimated to be free from collisions before the probe laser irradiation.

Figure 5 shows the rotational distributions of $\mathrm{NO}\left(v^{\prime}\right.$ $=0,1,2)$ obtained after the corrections described above. The squares and circles in Fig. 5 show the rotational populations of each spin-orbit and $\Lambda$-type sublevels. For each sublevel, fine irregularities of the population against $j^{\prime}$ remain even after the averaging of several scans and we do not consider these structures physically meaningful. The populations were derived from the $(0,0),(0,1)$, and $(0,2)$ bands, which cover all the rotational levels plotted in the figure. The validity of the correction procedure is confirmed by the fact that the populations derived from the $(1,0),(1,1)$, and $(2,2)$ bands are consistent with those shown in Fig. 5. The horizontal bars shown with arrows in the figure indicate the upper limits of the population of those levels for which we could not find any peak at the appropriate wavelengths. These limits were set to the largest undulation of the baseline. Thus the real populations might be lower than these limits. The total population of each vibrational state is normalized to appear in the same area by utilizing the Boltzmann fitting described below. In this study, the new and old NO products are not distinguished by isotopic labeling. The fractions of the old NO in the $v^{\prime}=0,1$, and 2 levels are estimated to be $77 \%, 62 \%$, and $50 \%$, respectively. ${ }^{14}$

Figure 5 shows that the global features of the rotational distributions in the three vibrational levels are similar and broad peaks are located at around $j^{\prime} \approx 60$. The populations calculated by the phase space theory (PST) $)^{35,36}$ are also shown by dotted lines. The PST distribution was obtained with the mean collision energy in our experimental condition $(52 \mathrm{~kJ} / \mathrm{mol})$ and by taking account of the centrifugal barrier in the exit region. In comparison with the PST distribution, the observed distribution can be characterized as follows. The distributions for the levels with low rotational quantum number $\left(j^{\prime}<50\right)$ are well reproduced by the statistical theory. The levels with middle $j^{\prime}\left(j^{\prime}=50-80\right)$ are more populated than predicted by the statistical theory and, in the high- $j^{\prime}$ region $\left(j^{\prime}=80-100\right)$, the observed populations drops more rapidly as $j^{\prime}$ increases than the statistical ones. There is no population in $j^{\prime}>100$. The statistical distribution for $\mathrm{NO}\left(v^{\prime}=0, j^{\prime}<50\right)$ was already observed by Tsurumaki et al. ${ }^{15}$ However, the extended measurement of this study up to the higher $j^{\prime}$ levels reveals that the rotational distributions of $\mathrm{NO}\left(v^{\prime}=0,1,2\right)$ gradually deviate from the statistical trend beyond $j^{\prime} \approx 50$ and completely differ from the PST predictions in the high- $j^{\prime}$ region $\left(j^{\prime}=80-100\right)$. The possible dynamical reasons for the decrease of high- $j^{\prime}$ population will be discussed in the rest of the paper.

Integrated populations and mean values of the observed distributions were calculated from simple analytical functions representing the distributions. Due to the sharp decrease in high- $j^{\prime}$ region, the distributions cannot be fitted to a single Boltzmann distribution function, nor a linear surprisal. Therefore, to fit the distributions, we adopted an effective functional form: two Boltzmann-type functions switched at a certain value $j_{c}$, i.e.,

$$
P\left(j^{\prime}\right)= \begin{cases}A_{1}\left(2 j^{\prime}+1\right) \exp \left(-E_{j^{\prime}} / k_{B} T_{1}\right) & \left(j^{\prime}<j_{c}\right), \\ A_{2}\left(2 j^{\prime}+1\right) \exp \left(-E_{j^{\prime}} / k_{B} T_{2}\right) & \left(j^{\prime}>j_{c}\right),\end{cases}
$$

where $E_{j}$ is the rotational energy and $k_{B}$ is the Boltzmann constant. In the least-squares fitting procedure, $A_{i}{ }^{\prime}$ s and $T_{i}{ }^{\prime} \mathrm{s}$ were determined for a given $j_{c}$. As an example, Fig. 6 shows the comparison of the observed and the fitted populations of $\mathrm{NO}\left(v^{\prime}=0,{ }^{2} \Pi_{3 / 2} A^{\prime}\right)$. Note that the form of Eq. (3) has no physical meaning related to the reaction dynamics and the 


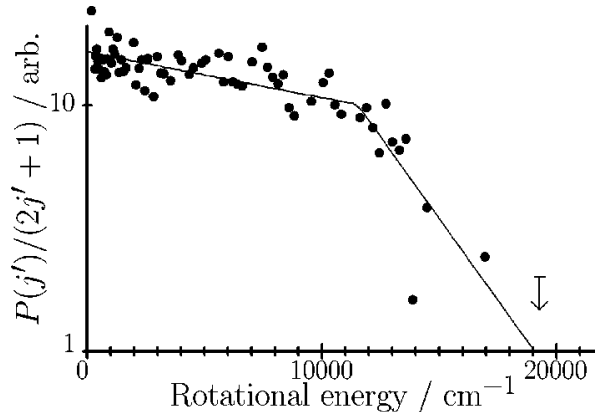

FIG. 6. Boltzmann plot of the rotational state distributions of $\mathrm{NO}\left(v^{\prime}=0\right.$, $\left.{ }^{2} \Pi_{3 / 2} A^{\prime}\right)$. Filled circles denote the observed populations. Solid lines are least-squares-fitted lines for the two Boltzmann-type functions switched at $j_{c}$ (see text).

fitting parameters $A_{i}$ 's and $T_{i}$ 's are only utilized to find the average of the scattered data and to interpolate the populations of those levels for which isolated rotational lines were not observed.

The results of the fitting are shown in Table I. Using these fitted populations, we were able to determine the mean rotational energies and the vibrational distribution, which are also shown in Table I. The vibrational distribution decreases with vibrational quantum number and the relative population agrees to the previous results shown by Akagi et al. ${ }^{14}$ although a slight difference can be seen. The ratio of the populations of $v^{\prime}=1$ and 2 obtained in this work rather agrees with that obtained by the recent time-resolved FTIR measurement of Hancock et al. ${ }^{17}$ The observed mean rotational energies are similar to those of PST, which may reflect the global statistical feature of the distribution.

It can be seen in Table I that the rotational excitation of the NO products found in this study is larger than those observed before. The main rotational temperature $T_{1}$ $=39000 \mathrm{~K}$ for $\mathrm{NO}\left(v^{\prime}=0,{ }^{2} \Pi_{1 / 2} A^{\prime}\right)$ is almost four times as high as the previous value for the same sublevel. ${ }^{15}$ Main reason of this difference is that the previous study ${ }^{15}$ estimated the rotational temperature as the lower limit rather than the least-squares-fitted value since the highest rotational energy of $j^{\prime} \approx 50\left(E_{\mathrm{rot}} \approx 4000 \mathrm{~cm}^{-1}\right)$ was insufficient to precisely determine the rotational temperature above $10000 \mathrm{~K}$. Furthermore, the present data set have much better quality than the previous one and the latter scatters around the former. We therefore conclude that the present result is consistent with the previous one and is much more reliable. For $\mathrm{NO}\left(v^{\prime}=1\right)$, the mean rotational energy is also 1.5 times higher than the previous reports. ${ }^{10,11}$ This discrepancy will be attributed to the collisional relaxation or lack of high- $j^{\prime}$ data in the previous measurement. The mean rotational energies of $\mathrm{NO}\left(v^{\prime}=1,2\right)$ obtained in this work are about 1.5 times higher than those estimated as the lower limits under the condition where 50 times more collisions are expected. ${ }^{17}$ As described above, the rotational distributions are close to the statistical distribution to some extent and the rotational temperature $\approx 20000 \mathrm{~K}$ is not extraordinarily high compared with the large exothermicity of this reaction.

As for the fine state dependence of the population, the difference between the two $\Lambda$-doublet states, $\Pi\left(A^{\prime}\right)$ and $\Pi\left(A^{\prime \prime}\right)$, is discernible and a slight preference for $\Pi\left(A^{\prime}\right)$ can be seen in Table I. As can be recognized from Fig. 5, this difference is not so obvious in low $j^{\prime}$ levels. Little preference for one of the $\Lambda$-type doublet components indicates that the motion of the intermediate is not restricted in a plane. This trend is consistent with the observation of the isotropic angular distribution of product rotational angular momentum relative to the scattering plane reported for $j^{\prime}=34.5$ by Tsurumaki et al. ${ }^{15}$ However, the preference for $\Pi\left(A^{\prime}\right)$ in the high- $j^{\prime}$ products may indicate the dominance of the in-plane torque to generate highly rotating products. On the other hand, the difference between the two spin-orbit components, $F_{1}$ and $F_{2}$ levels, is not significant for any $j^{\prime}$ level.

As recognized from Figs. 5 and 6, the peculiar feature of the rotational population of all the observed vibrational levels is the sharp decrease of the population in the high- $j^{\prime}$ region $\left(j^{\prime}=80-100\right)$. The possible factors giving rise to such distribution as an artifact are the predissociation above

TABLE I. Rotational temperatures, mean rotational energies and populations of NO.

\begin{tabular}{|c|c|c|c|c|c|}
\hline & $j_{c}{ }^{\mathrm{a}}$ & $T_{1} / \mathrm{K}^{\mathrm{a}}$ & $T_{2} / \mathrm{K}^{\mathrm{a}}$ & $\left\langle E_{\mathrm{rot}}\right\rangle / \mathrm{cm}^{-1}$ & Population $^{\mathrm{b}}$ \\
\hline$v^{\prime}=0,{ }^{2} \Pi_{1 / 2} A^{\prime}$ & 81.5 & $39000 \pm 7900\left(10000^{c}\right)$ & $11000 \pm 15100$ & 8300 & 0.51 \\
\hline$v^{\prime}=0,{ }^{2} \Pi_{1 / 2} A^{\prime \prime}$ & 76.5 & $20000 \pm 3400$ & $8400 \pm 3800$ & 7000 & 0.40 \\
\hline$v^{\prime}=0,{ }^{2} \Pi_{3 / 2} A^{\prime}$ & 86.5 & $34000 \pm 4400$ & $4700 \pm 4600$ & 7100 & 0.43 \\
\hline$v^{\prime}=0,{ }^{2} \Pi_{3 / 2} A^{\prime \prime}$ & 78.5 & $20000 \pm 1800$ & $7200 \pm 3900$ & 7300 & 0.38 \\
\hline$v^{\prime}=0$, total & & $28000 \pm 1900$ & & $7500\left[7200^{\mathrm{d}}\right]$ & $1.73\left(1.35^{\mathrm{e}}\right)$ \\
\hline$v^{\prime}=1,{ }^{2} \Pi_{1 / 2} A^{\prime}$ & 69.5 & $18000 \pm 1800$ & $6500 \pm 1900$ & 6000 & 0.28 \\
\hline$v^{\prime}=1,{ }^{2} \Pi_{1 / 2} A^{\prime \prime}$ & 65.5 & $18000 \pm 2800$ & $7900 \pm 2900$ & 6100 & 0.23 \\
\hline$v^{\prime}=1,{ }^{2} \Pi_{3 / 2} A^{\prime}$ & 74.5 & $19000 \pm 1800$ & $6700 \pm 2600$ & 6600 & 0.28 \\
\hline$v^{\prime}=1,{ }^{2} \Pi_{3 / 2} A^{\prime \prime}$ & 66.5 & $22000 \pm 3100$ & $8800 \pm 3300$ & 6500 & 0.22 \\
\hline$v^{\prime}=1$, total & & $20000 \pm 1100$ & & $6300\left(4000^{f}\right)\left[6900^{d}\right]$ & 1.00 \\
\hline$v^{\prime}=2,{ }^{2} \Pi_{1 / 2} A^{\prime}$ & 74.5 & $20000 \pm 1700$ & $8300 \pm 5200$ & 6500 & 0.22 \\
\hline$v^{\prime}=2,{ }^{2} \Pi_{1 / 2} A^{\prime \prime}$ & 62.5 & $20000 \pm 3700$ & $8100 \pm 3100$ & 6200 & 0.17 \\
\hline$v^{\prime}=2,{ }^{2} \Pi_{3 / 2} A^{\prime}$ & 67.5 & $23000 \pm 3500$ & $13300 \pm 6700$ & 7700 & 0.21 \\
\hline$v^{\prime}=2,{ }^{2} \Pi_{3 / 2} A^{\prime \prime}$ & 67.5 & $14000 \pm 1900$ & $8100 \pm 2300$ & 6400 & 0.16 \\
\hline$v^{\prime}=2$, total & & $20000 \pm 1400$ & & $6800\left[6500^{\mathrm{d}}\right]$ & $0.76\left(0.65,^{\mathrm{e}} 0.80^{\mathrm{g}}\right)$ \\
\hline
\end{tabular}

${ }^{a}$ The meanings of the fitting parameters are found in text.

${ }^{b}$ Normalized to $v^{\prime}=1$ population.

${ }^{\mathrm{c}}$ Tsurumaki et al. (Ref. 15).

${ }^{\mathrm{d}}$ Calculated using PST.

${ }^{\mathrm{e}}$ Akagi et al. (Refs. 13 and 14).
${ }^{\mathrm{f} B r o u a r d}$ et al. (Refs. 10 and 11).
${ }^{\mathrm{g}}$ Hancock et al. (Ref. 17). 
a threshold level and the neglect of the correction for detection efficiency. Since we carefully inspected such effects and made the proper correction as described above, we conclude that the sharp decrease of the population in the high- $j^{\prime}$ levels is the genuine one.

\section{B. Half-collision calculation: Modified statistical approach}

The observed sharp cutoffs in the product state distribution may invoke the idea that a kinematic effect such as angular momentum conservation or truncation of the impact parameters limits the production of high- $j^{\prime}$ products. In order to check such effects, we performed the PST calculations with the maximum total angular momenta $J_{\max }=100,220$, and 330, where 100 is the minimum value estimated from the experimental rate constant, 220 corresponds to the impact parameter where the opacity function becomes negligible in our full-collision QCT result, and 330 is 1.5 times of that value. The calculated distributions did not change significantly with the maximum total angular momentum. Therefore we can state that the decrease of the high- $j$ ' populations is not due to this type of kinematic effect.

We adopted a statistical approach to reproduce the sharp decrease of the population in high- $j^{\prime}$ levels since the overall trends of the observed rotational distributions were close to those of the PST prediction. In this approach, statistical adiabatic channel model (SACM), ${ }^{37}$ which is a modified version of PST, is a suggestive concept. It is well known that the population of high- $j^{\prime}$ products calculated by SACM tends to be depressed compared with that calculated by PST ${ }^{38}$ In short, this depression results from the difference of the potential of free products and reaction intermediate. It can be expected that this effect causes the observed sharp decrease in the high- $j^{\prime}$ products in the $\mathrm{O}\left({ }^{1} \mathrm{D}\right)+\mathrm{N}_{2} \mathrm{O}$ reaction. However, SACM assumes that the system remains on the same adiabatic potential curve as going from the intermediate to the product, which means that the product molecules need to have sufficiently small velocities. It is questionable whether this condition is satisfied in the $\mathrm{O}\left({ }^{1} \mathrm{D}\right)+\mathrm{N}_{2} \mathrm{O}$ reaction since the average departure velocity of the product NO is large due to the high exothermicity. ${ }^{15}$

Recently, Larregaray et al. ${ }^{39}$ combined the statistical assumption for the intermediate and the interactions in the exit potential, to correct the statistical treatment. They analyzed the exit channel dynamics of unimolecular reaction of simple tri-atomic systems. Once they assumed the statistical ensemble at the transition state, they solved the equations of motion without any adiabatic assumption on an analytically modeled PES with a harmonic potential whose curvature decreases exponentially with the distance between the products. Their result was that even if the statistical distribution was achieved at the reaction intermediate, the trajectories starting from this statistical intermediate resulted in less high- $j^{\prime}$ population at the products than the PST prediction. This high- $j^{\prime}$ depression is generated by the rotationtranslation interaction in the exit region of PES. Although the ground for the statistical distribution at the intermediate in our system is not trivial and can be different from that in Larregaray et al.'s one, the concept of the exit region inter-
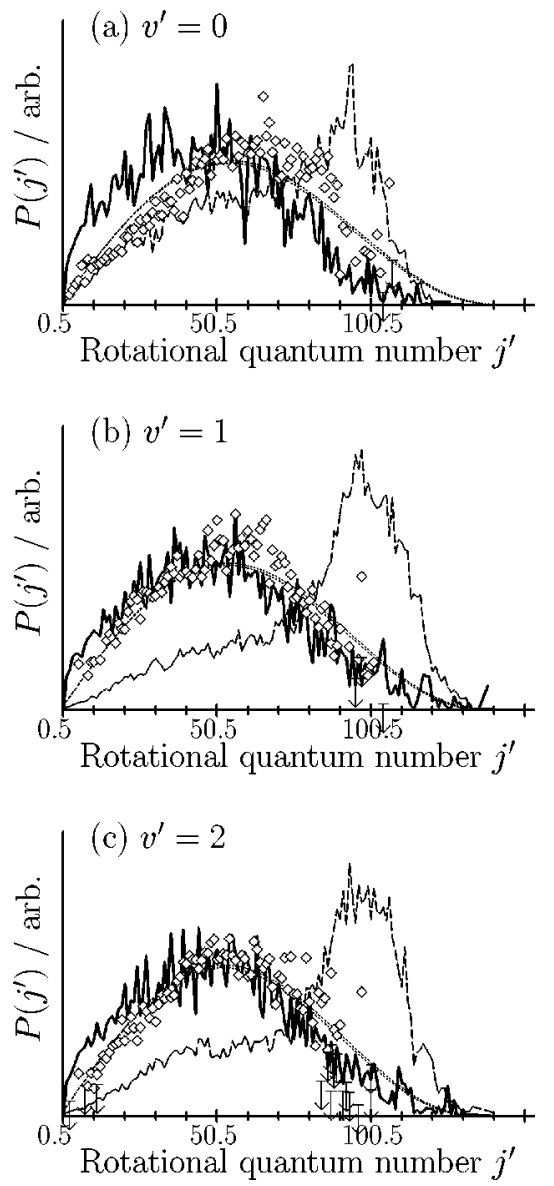

FIG. 7. Comparison of observed and calculated rotational state distributions of $\mathrm{NO}\left(v^{\prime}=0,1,2\right)$. Squares denote the observed population averaged for all the spin-orbit and $\Lambda$-type sublevels. Dotted, bold, and dashed lines are calculated distributions of PST, half-collision QCT, and full-collision QCT (see text), respectively.

action modifying the statistical distribution to reduce high- $j^{\prime}$ populations may be applicable to the present case.

Stimulated by these statistical approaches, we performed a kind of QCT calculation which assumes statistical distribution at the intermediate and propagates the trajectory en route to the products on the $a b$ initio surface calculated by Takayanagi et al. ${ }^{6-8}$ We call this calculation "half-collision QCT calculation." The concept of this calculation may have a lot in common with SACM and the work of Larregaray et al. ${ }^{39}$ concerning the exit region interaction, but it does not depend on adiabatic assumption or analytical model of the potential. The results of the half-collision calculations are shown by bold lines in Fig. 7. In the figure, the observed distributions are averaged for all the four sublevels of ${ }^{2} \Pi$ states for clarity. The decrease of the high- $j^{\prime}$ population is well reproduced in the half-collision calculation. This indicates that the interaction in the exit region of PES indeed has an effect of yielding small high- $j^{\prime}$ populations as is pointed out in Ref. 39.

The effect of high- $j{ }^{\prime}$ depression due to the exit region interaction can be understood by considering the trajectories in the phase space. Rather than the trajectories propagating from the intermediate to the products, time-reversed trajectories which start from the product NO's and enter into the intermediate are better for describing the exit interaction ef- 


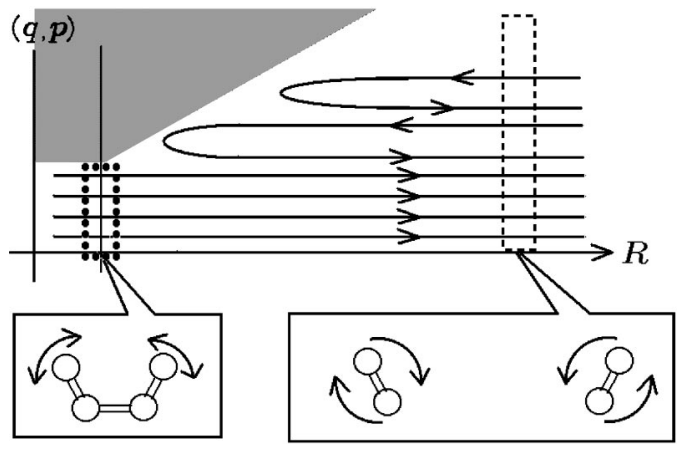

FIG. 8. Schematic figure of the phase space of the reaction system. The horizontal axis represents the distance $R$ between two NO's. The vertical axis represents the other degrees of freedom such as bending motions. The points in the gray area are not accessible with the available energy of the reaction due to the large vibrational potential energy. The inaccessible region becomes larger as $R$ decreases and the rotational motions of product NO's change into bending and torsional vibrations in the intermediate. The "statistical distribution of the intermediate" populates all the phase space points rounded by the dotted lines equally, while the statistical distribution of the products such as PST distribution populates all the points within the dashed lines.

fect. The use of time-reversed trajectories is certificated by the fact that the classical equations of motion are symmetric with respect to the time reversal. Figure 8 schematically represents the phase space of the system where the horizontal axis denotes the distance $R$ between the CM of two NO moieties and the vertical axis represents the rotational degrees of freedom. Since the free rotational motion of NO's in $R=\infty$ correlates mostly to the bending and torsional vibrations for finite $R$, certain regions of the phase space are not accessible due to the bending and torsional potential energy which becomes higher as $R$ decreases. Then we see that some of the trajectories are reflected due to this $R$-dependent vibrational potential. In other words, the product states corresponding to these trajectories cannot be produced by the reaction. If the product states have high rotational energy, the translational energy will be too small to surmount the vibrational potential and hence such trajectories have large probability to be reflected. This means that the statistical distribution at the intermediate corresponds to distribution with depressed high- $j^{\prime}$ populations when propagated to the products.

The essential point for the exit interaction effect of depressing the high- $j$ ' populations in the products is the difference between "the statistical distribution of the intermediate" and that of the products. In Fig. 8, the accessible region at the intermediate is rounded by dotted lines and that in the product by dashed lines. If the intermediate has the statistical distribution, all the phase space points within the dotted lines are equally populated. On the other hand, the statistical distribution of the products, which is calculated with the PST formalism, populates all the phase space points within the dashed lines. Since there exist reflected trajectories due to the vibrational potential, the statistical distribution at the intermediate does not yield statistical distribution in the products.

Despite the improvement of reproducing the sharp decrease in the half-collision calculation, the calculated distributions are a little biased to the low $-j^{\prime}$ region compared to the observed ones. This difference can be explained in terms of the following two factors, the accuracy of the PES and the validity of the statistical assumption at the intermediate. If the statistical assumption works well in describing the real distribution of the intermediate, the present PES is inaccurate and overestimates the depression of the rotational excitation of the products in the exit region. On the other hand, if the extent of the depression of the rotational excitation in the exit region of the present PES is correct, the statistical assumption is invalid. In this case, the real distribution of the intermediate is more biased toward the phase space points which produce high- $j$ ' products than the statistical one. These phase space points can be regarded as those with high bending and/or torsional vibrations since the NO rotation correlates approximately to the bending and torsional vibrations in the ONNO intermediate.

\section{Reaction dynamics}

The validity of the statistical assumption on the current PES can be discussed from the comparison between the results of the half-collision calculation and those of the fullcollision calculation. The rotational distributions obtained by the full-collision calculations are shown in Fig. 7 as dashed lines. The distributions are too much rotationally excited for all the vibrational levels and the agreement with the experimental result is poor. From the failure of the full-collision calculation, we learn two things: First, the present global PES cannot be considered accurate enough to describe the product state distribution when it is used in full-collision calculations. Second, the reaction intermediate on this PES tends to be too much biased toward the phase space points yielding high- $j^{\prime}$ products. Although the accuracy of the current PES is not perfect, we consider that the success of the half-collision calculation still has its significance from the following reason. The half-collision calculation starts with the structure which is rather similar to the two independent NO fragments since the two NO's are quite distant at 2.263 $\AA$. Furthermore, we found that only negligible amount of trajectories went back to shorter distance than the initial value. Thus, the trajectories in the half-collision calculation experience rather limited portion of the global PES than is imagined from the word "half," although we used this word for simplicity. Because there is only small anisotropy of the PES in the exit region except for the hard-core repulsion of the nuclei, we consider that the results of the half-collision calculation are not very sensitive to the details of the PES. Therefore, if the inaccuracy of the exit region of the present PES is not so severe, the success of the half-collision calculation indicates that the statistical assumption for the intermediate represent the real system to some extent.

As stated in Sec. IV B, the near-statistical distribution with the sharp decrease of the high- $j^{\prime}$ populations can be explained by the balance between the distribution at the ONNO intermediate and the interaction in the exit region of the PES. Since no deep well exists in the PES of this reaction, it is plausible that the reaction intermediate does not have the completely statistical distribution. Although the full-collision calculation hardly reproduced the observed distributions, the calculated result indicates that the intermediate is biased toward the phase space points producing high- $j^{\prime}$ 
products. This trend is qualitatively consistent with the bias to make the half-collision result closer to the experimental one. Therefore, we suggest that the real dynamics of this reaction consists of the following two factors: (i) an ONNO intermediate with near-statistical distribution a little biased toward the phase space points correlating with high- $j^{\prime}$ products; (ii) the rotation-translation interaction in the exit region similar to the present PES, which makes this nearstatistical intermediate distribution correspond to the experimentally observed high- $j^{\prime}$ depressed distribution of the products.

The occurrence of the near-statistical distribution in this highly exothermic reaction without a stable intermediate is not expected in the traditional understanding of gas-phase chemical reaction dynamics. As an important factor which causes the considerable energy randomization in the ONNO intermediate, Akagi et al. ${ }^{21}$ suggested mass effects. According to their discussions, the presence of four heavy atoms in the system provides low frequency vibrations to increase the state density and also causes large momentum coupling among the local vibrational modes. These effects seem to occur even in the full-collision calculation on the current PES since the calculated vibrational and rotational state distributions of new and old NO's are very similar. ${ }^{40}$ However, the discrepancy between the full-collision calculation and experimental observation should be attributed to the defect of the current PES since the full-collision calculation already includes the mass effects. It is found that the vibrational distribution obtained by the full-collision calculation ${ }^{40}$ is less excited than the experimentally observed one, ${ }^{14}$ in contrast to the overestimate of the rotational excitation. We therefore consider that poorly reproduced couplings between the stretching motions and bending/torsional motions of the ONNO system are the possible defect of the current PES.

In closing this paper, it should be emphasized that the comparison between the half-collision and the full-collision calculations is very helpful to deepen the understanding of what takes place on a PES during the reaction. This approach will be promising when a more accurate PES reproducing the product state distribution of this reaction is obtained.

\section{SUMMARY AND CONCLUSIONS}

We have determined the nascent product state distributions of $\mathrm{NO}\left(X^{2} \Pi, v^{\prime}=0-2 ; j^{\prime}\right)$ produced from the reaction $\mathrm{O}\left({ }^{1} \mathrm{D}\right)+\mathrm{N}_{2} \mathrm{O}$ up to $j^{\prime} \approx 100$ where the distribution decayed under the noise level and no significant populations were found in higher rotational levels. All the rotational state distributions in these vibrational levels are similar and near statistical. However, the populations in high- $j^{\prime}$ levels decreased more rapidly than PST prediction. This behavior can be understood in view of the rotation-translation interaction in the exit region of the PES. The depression of the high- $j^{\prime}$ population was reproduced in half-collision QCT calculations with the assumption of statistical distribution at the intermediate. We concluded therefore that near-statistical distribution a little biased toward the high- $j^{\prime}$ products is generated at the intermediate and then this distribution is propagated to yield high- $j^{\prime}$ depressed product distribution due to the exit region interaction.

\section{ACKNOWLEDGMENTS}

The authors are grateful to H. Tamada for assistance in the measurement. This research was partially supported by the Japan Society for the Promotion of Science, Grant-in-Aid for Scientific Research, 13640511, 2001-2002.

${ }^{1}$ D. L. Baulch, R. A. Cox, R. F. Hampson, Jr., J. A. Kerr, J. Troe, and R. T. Watson, J. Phys. Chem. Ref. Data 9, 295 (1980).

${ }^{2}$ R. Atkinson, D. L. Baulch, R. A. Cox, R. F. Hampson, Jr., J. A. Kerr, and J. Troe, J. Phys. Chem. Ref. Data 21, 1125 (1992).

${ }^{3}$ J. R. Hetzler, M. P. Casassa, and D. S. King, J. Phys. Chem. 95, 8086 (1991).

${ }^{4}$ I. Last, A. Aguilar, R. Sayós, M. González, and M. Gilibert, J. Phys. Chem. A 101, 1206 (1997).

${ }^{5}$ M. González, R. Valero, J. M. Anglada, and R. Sayós, J. Chem. Phys. 115, 7015 (2001).

${ }^{6}$ H. Akagi, A. Yokoyama, Y. Fujimura, and T. Takayanagi, Chem. Phys. Lett. 324, 423 (2000).

${ }^{7}$ T. Takayanagi and A. Wada, Chem. Phys. 269, 37 (2001).

${ }^{8}$ T. Takayanagi and H. Akagi, Chem. Phys. Lett. 363, 298 (2002).

${ }^{9}$ K. Honma, Y. Fujimura, O. Kajimoto, and G. Inoue, J. Chem. Phys. 88, 4739 (1988).

${ }^{10}$ M. Brouard, S. P. Duxon, P. A. Enriquez, R. Sayos, and J. P. Simons, J. Phys. Chem. 95, 8169 (1991).

${ }^{11}$ M. Brouard, S. P. Duxon, P. A. Enriquez, and J. P. Simons, J. Chem. Phys. 97, 7414 (1992).

${ }^{12}$ X. Wang, H. Li, Q. Zhu, F. Kong, and H. Yu, J. Chin. Chem. Soc. (Taipei) 42, 399 (1995).

${ }^{13}$ H. Akagi, Y. Fujimura, and O. Kajimoto, J. Chem. Soc., Faraday Trans. 94, 1575 (1998).

${ }^{14}$ H. Akagi, Y. Fujimura, and O. Kajimoto, J. Chem. Phys. 111, 115 (1999).

${ }^{15}$ H. Tsurumaki, Y. Fujimura, and O. Kajimoto, J. Chem. Phys. 111, 592 (1999)

${ }^{16}$ P. J. Pisano, M. S. Westley, and P. L. Houston, Chem. Phys. Lett. 318, 385 (2000).

${ }^{17}$ G. Hancock and V. Haverd, Phys. Chem. Chem. Phys. 5, 2369 (2003).

${ }^{18}$ C. B. Cleveland and J. R. Wiesenfeld, J. Chem. Phys. 96, 248 (1992).

${ }^{19}$ D. G. Sauder, J. C. Stephenson, D. S. King, and M. P. Casassa, J. Chem. Phys. 97, 952 (1992).

${ }^{20}$ R. Sayós, C. Oliva, and M. González, J. Chem. Phys. 113, 6736 (2000).

${ }^{21}$ H. Akagi, Y. Fujimura, and O. Kajimoto, J. Chem. Phys. 110, 7264 (1999).

${ }^{22}$ N. Goledstein, G. D. Greenblatt, and J. R. Wiesenfeld, Chem. Phys. Lett. 96, 410 (1983).

${ }^{23}$ T. Takayanagi, Chem. Phys. (to be published).

${ }^{24}$ S. F. Adams, C. A. DeJoseph, Jr., C. C. Carter, T. A. Miller, and J. M. Williamson, J. Phys. Chem. A 105, 5977 (2001).

${ }^{25}$ G. D. Greenblatt and A. R. Ravishankara, J. Geophys. Res. 95, 3539 (1990).

${ }^{26}$ J. Luque and D. R. Crosley, J. Chem. Phys. 112, 9411 (2000).

${ }^{27}$ W. H. Press, S. A. Teukolsky, W. T. Vetterling, and B. P. Flannery, Numerical Recipes in C (Cambridge University Press, Cambridge, 1992).

${ }^{28}$ A. R. W. McKellar, J. K. G. Watson, and B. J. Howard, Mol. Phys. 86, 273 (1995).

${ }^{29}$ Y. Fujimura, H. Tsurumaki, and O. Kajimoto, Bull. Chem. Soc. Jpn. 75, 2309 (2002).

${ }^{30}$ P. Felder, B.-M. Haas, and J. R. Huber, Chem. Phys. Lett. 186, 177 (1991).

${ }^{31}$ J. Danielak, U. Domin, R. Kȩpa, M. Rytel, and M. Zachwieja, J. Mol. Spectrosc. 181, 394 (1997).

${ }^{32}$ R. Altkorn and R. N. Zare, Annu. Rev. Phys. Chem. 35, 265 (1984).

${ }^{33}$ J. Luque and D. R. Crosley, J. Chem. Phys. 111, 7405 (1999).

${ }^{34}$ M. Islam, I. W. M. Smith, and J. W. Wiebrecht, J. Phys. Chem. 98, 9285 (1994).

${ }^{35}$ P. Pechukas and J. C. Light, J. Chem. Phys. 42, 3281 (1965).

${ }^{36}$ J. C. Light, Discuss. Faraday Soc. 44, 14 (1967).

${ }^{37}$ M. Quack and J. Troe, Ber. Bunsenges. Phys. Chem. 78, 240 (1974).

${ }^{38}$ T. M. Ticich, T. R. Rizzo, H.-R. Dübal, and F. F. Crim, J. Chem. Phys. 84, 1508 (1986)

${ }^{39}$ P. Larregaray, L. Bonnet, and J. C. Rayez, J. Chem. Phys. 114, 3349 (2001)

${ }^{40}$ S. Kawai, Y. Fujimura, O. Kajimoto, and T. Takayanagi (unpublished). 